\title{
Semantic validation of the use of SNOMED CT in HL7 clinical documents
}

Stijn Heymans, Matthew McKennirey and Joshua Phillips

* Correspondence: stijn. heymans@semanticbits.com SemanticBits, LLC, 13921 Park Center Road Suite 420, Herndon, VA 20171, USA

\begin{abstract}
Background: The HL7 Clinical Document Architecture (CDA) constrains the HL7 Reference Information model (RIM) to specify the format of HL7-compliant clinical documents, dubbed CDA documents. The use of clinical terminologies such as SNOMED $C T^{\circledR}$ further improves interoperability as they provide a shared understanding of concepts used in clinical documents. However, despite the use of the RIM and of shared terminologies such as SNOMED $C T^{\circledR}$, gaps remain as to how to use both the RIM and SNOMED $C T^{\circledR}$ in HL7 clinical documents. The HL7 implementation guide on Using SNOMED CT in HL7 Version 3 is an effort to close this gap. It is, however, a human-readable document that is not suited for automatic processing. As such, health care professionals designing clinical documents need to ensure validity of documents manually.
\end{abstract}

Results: We represent the CDA using the Ontology Web Language OWL and further use the OWL version of SNOMED $C T^{\mathbb{R}}$ to enable the translation of CDA documents to so-called OWL ontologies. We formalize a subset of the constraints in the implementation guide on Using SNOMED CT in HL7 Version 3 as OWL Integrity Constraints and show that we can automatically validate CDA documents using OWL reasoners such as Pellet. Finally, we evaluate our approach via a prototype implementation that plugs in the Open Health Workbench.

Conclusions: We present a methodology to automatically check the validity of CDA documents which make reference to SNOMED $C T^{\circledR}$ terminology. The methodology relies on semantic technologies such as OWL. As such it removes the burden from IT health care professionals of having to manually implement such guidelines in systems that use HL7 Version 3 documents.

\section{Background} Introduction

Health Level Seven International (HL7) [1] is a non-profit organization that develops standards to increase the interoperability of health care information technology. One such standard is the Reference Information Model (RIM) [2] that functions as the common information model for all further specified information models and messages developed under the auspices of HL7. For example, the HL7 standard for writing clinical documents is provided by the Clinical Document Architecture (CDA) [3] and is a constraining of the RIM. It specifies how HL7 clinical documents should be structured while using classes and attributes defined in the RIM. 
The advantage of using such a layered architecture of models for different purposes is its provision of syntactic (and to a certain extent semantic) interoperability throughout the health care domain. Clinical documents for exchange between health care professionals that satisfy this CDA (CDA documents), are syntactically understandable by both ends of the exchange. The CDA caters for some semantic interoperability. Indeed, it prescribes what meta-vocabulary to use in the form of directions such as to use the class SubstanceAdministration for administering a substance. However, an extra factor is needed to understand also the particular data being sent in such clinical documents.

Clinical terminologies such as SNOMED $\mathrm{CT}^{\circledR}$ provide the means for standardizing such data. If a health care professional references a CDA SubstanceAdministration of a SNOMED $\mathrm{CT}^{\circledR}$ concept with ID 433181003 , the receiving professional knows that Amoxicillin $775 \mathrm{mg}$ extended release tablet is targeted for SubstanceAdministration. Note that such clarity (or semantic interoperability) is important for humans, but becomes an even more pressing issue if the clinical documents are to form the basis of automated decision making: is the substance administration of Amoxicillin appropriate given the exhibited symptoms?

Even though a combination of the HL7 RIM and the use of standardized clinical terminologies goes a long way toward semantic interoperability, exactly their use together can lead to interoperability problems. Indeed, there is a certain overlap between the semantics of the RIM and the semantics of SNOMED CT ${ }^{\circledR}$ codes. A SNOMED CT ${ }^{\circledR}$ code may express a meaning that the RIM expresses using a combination of classes [4].

To clarify the use of SNOMED CT ${ }^{\mathbb{B}}$ in the context of HL7 documents, guidelines are established in [4]. The guidelines indicate, among others, what the code of a certain CDA Observation should be if a SNOMED $\mathrm{CT}^{\circledR}$ concept is used (it should be a subtype of the SNOMED CT ${ }^{\circledR}$ concept Observable entity). These guidelines are written in natural language and at present no automated means is available to check whether a clinical document satisfies them to ensure that both sender and receiver can interpret the result the same way.

The goal of this work is to verify how to automatically validate CDA documents for satisfaction of the guidelines on the use of SNOMED CT ${ }^{\circledR}$ in HL7 documents.

We provide a possible solution for this problem by using the Web Ontology Language OWL [5]. OWL is a W3C standard for representing knowledge on the Web, and serves in general as a language for expressing ontologies, where ontologies were conceived as formalized representations of knowledge that provide a "shared understanding" [6] of certain domains. Moreover, one can reason over representations in OWL: one can determine which concepts are equivalent, which are subsumed, and whether the knowledge base is inconsistent. As such it is an excellent candidate for a lingua franca in the health care domain, and able to inject the necessary automatization for semantic interoperability. We show that by writing the available knowledge in the current scenario in OWL and by using OWL reasoners such as Pellet, we can exactly tackle the question does my CDA document satisfy the guidelines on the use of SNOMED CT ${ }^{\circledR}$ ?

This article reports on how to write OWL representations of the different knowledge involved in the above problem when dealing with CDA documents. We define an ontology for the CDA, use the OWL version of SNOMED CT ${ }^{\circledR}$, write a specific subset of the guidelines (the SNOMED $\mathrm{CT}^{\circledR}$ vocabulary domain constraints [[4], Section 5]) 
as OWL, and show how to transform CDA documents to OWL ontologies. We then indicate how to use automated reasoning via ontology reasoners to validate CDA documents for conformance to the usage of SNOMED $\mathrm{CT}^{\circledR}$. We further report on a prototype implementation that extends the Open Health Workbench [7] with a plug-in for such semantic validation. Finally, we discuss some common errors that we found in CDA documents with respect to to the SNOMED $\mathrm{CT}^{\circledR}$ guidelines and we identify areas of future work.

\section{Methods}

\section{Reasoning with OWL Integrity Constraints}

One of the corner stone ideas of the envisioned Semantic Web [8] is to move from human-readable content on the Web to machine-processable content that can be automatically reasoned about. Layered on top of basic Web technologies such as XML, RDF [9], RDF(S) [10], the OWL Web Ontology Language [5], and its expressive successor OWL 2 [11] provide for an expressive logic-based representation of knowledge on the Web.

Expressive fragments of OWL, the so-called $O W L D L$ fragments, correspond directly to a particular Description Logic [12]. Traditionally, Description Logics form a set of logical languages that balance complexity and expressiveness: they are usually decidable fragments of undecidable first-order logic with a syntax that has as basic building blocks concepts and relationships or roles. In contrast with first-order logic, they allow for decidable reasoning and can handle tasks such as verifying whether one concept is subsumed by another concept or whether concepts are satisfiable.

As such, Description Logics are recognized also in the health care domain as a useful framework to build clinical terminologies. SNOMED CT ${ }^{\circledR}$ (see below) is defined as a particular Description Logic theory, allowing users to, for example, identify redundancy by querying for clinical concepts that are equivalent [13].

We use in this article the Manchester OWL syntax [14] as it is easy for humans to read and close to the underlying Description Logics syntax. we refrain from defining the syntax in detail but will explain its intuition whenever we use it. Take the following example:

\section{PCNAllergy SubClassOf \\ (causativeAgent some Penicillin)}

The basic building blocks are the concepts PCNAllergy and Penicillin which are used to classify individuals and their type. The basic relationship or role used is causativeAgent which relates individuals to other individuals, namely its causative agents. The concept expression (causativeAgent some Penicillin) is called an exists restriction as it captures all individuals that have some causative agent that is a penicillin. Axiom (1) then imposes that every individual that is of type PCNAllergy also needs to have a causativeAgent relation with some individual that is of type Penicillin.

We can make this axiom stronger by writing:

\section{PCNAllergy EquivalentTo}

Allergy and (causativeAgent some Penicillin) 
Instead of a SubClass Of axiom this is an EquivalentTo axiom and can be seen as 2 SubClassOf axioms: axiom (1) and $\begin{array}{ll}\text { (Allergy and } & \text { (causativeAgent some Penicillin)) } \\ \text { SubClassof } & \text { PCNAllergy }\end{array}$

It indicates that individuals of type PCNAllergy are exactly those allergies that have a causative agent that is penicillin.

The semantics of such sets of OWL axioms, called ontologies, is given by first-order interpretations and thus obeys the open world assumption. This open world assumption has important consequences on the type of reasoning possible with OWL. Assume we have a particular individual $i$ that is of type PCNAllergy. Denoted in traditional first-order logic notation, we have PCNAllergy (i). With the SubClassOf axiom (1), we deduce that there exists some individual $j$ such that causativeAgent $(i, j)$ and Penicillin (j) holds. Given this open world semantics, if such a $j$ does not exist explicitly, it is assumed to exist implicitly to satisfy the axiom. In the case of reasoning with domain knowledge such as clinical terminologies such standard logical entailment allows to answer questions such as "When my patient has an allergy, should this be associated with a causative agent?" The usual semantics says "yes, there is some causative agent."

In the context of pure conceptual reasoning such an open world assumption is suitable and desirable (one does not want to include a representative data set to test equivalence of two concepts). However, when data is present that needs to conform to an ontology, one would like the axioms to behave more like integrity constraints (ICs), i.e., one would like to treat OWL as a schema language for the instance data. In the above example, a data set containing only PCNAllergy(i) should lead to a violation of the PCNAllergy axiom. A data set containing on the other hand PCNAllergy $(i)$, causativeA$\operatorname{gent}(i, j)$ and Penicillin $(j)$ is not violating the axiom. Indeed, whereas logical entailment is useful for reasoning over the domain knowledge, when dealing with clinical documents, one does not just want to know that there is a sole, possibly unknown, causative agent. One wants to verify that the clinical document is explicitly mentioning this causative agent.

In [15] such an integrity constraint semantics for OWL axioms is defined, enabling OWL axioms to be used as constraints that must be satisfied by particular instance data, thus effectively implementing a closed world assumption. An implication for our treatment of CDA documents is that, whereas the usual semantics of OWL is interested in what can be inferred, the integrity constraint semantics cares about what is or is not in the document. A prototype integrity constraint validator, Pellet-ICV [16], is implemented based on the Pellet OWL reasoner [17].

\section{SNOMED CT}

The Systematized Nomenclature of Medicine - Clinical Terms (SNOMED CT ${ }^{\circledR}$ ) [18] is a reference terminology for clinical data. Such a clinical reference terminology is described by [13] as

"[. . .] a set of concepts and relationships that provides a common reference point for comparison and aggregation of data about the entire health care process, recorded by multiple different individuals, systems, or institutions. The main purpose of a reference terminology for clinical data is the retrieval and analysis of data 
relating to the causes of disease, the treatment of patients, and the outcomes of the overall health care process."

The work described in this paper uses the SNOMED CT ${ }^{\circledR}$ core International Release of January 2010 [18], which consists of 291144 concepts. For example, the concept Amoxicillin $775 \mathrm{mg}$ extended release tablet (product) has a concept identifier 433181003 and is indicated by SNOMED CT ${ }^{\circledR}$ to be a 350162003 |Oral form Amoxicillin (product) where we use the conceptId |Fully Specified Name format for SNOMED $\mathrm{CT}^{\circledR}$ concepts. Moreover, it has as an active ingredient the concept 372687004 |Amoxicillin (substance). Note that also relationships between concepts have an identifier: has active ingredient has the identifier 127489000 . For readability, we will refrain from using SNOMED $\mathrm{CT}^{\circledR}$ concept identifiers or fully specified names. Instead, we will use short human-readable names throughout the paper where appropriate.

Such stated relationships of SNOMED $\mathrm{CT}^{\circledR}$, i.e., the clinical statements that are directly defined by authors or editors, in contrast with inferred relationships, can be translated to the ontology language OWL to enable specific inferencing such as determining the equivalence of concepts or hierarchical relationships between concepts. The SNOMED CT Stated Relationships Guide [19] describes a script that provides exactly this translation.

In Table 1, we extend the above example that shows how SNOMED CT ${ }^{\mathbb{R}}$ indicates that Amoxicillin is a particular Penicillin and that an Allergy to penicillin has Penicillin as a causative agent.

The equivalent OWL representation of Table 1 is listed in Table 2. The IsA relations are directly translated using the SubClassOf construct of OWL, while other relationships are defined using OWL exists restrictions: the expression (HasActiveIngredient some Amoxicillin) collects all individuals that have some active ingredient that is a Amoxicillin. Axiom (1) in Table 2 then indicates that every individual that is a AmoxicillinTablet is also an individual that has some active ingredient that is a Amoxicillin.

The only non-trivial axiom is axiom (4) that uses the special non-SNOMED $\mathrm{CT}^{\circledR}$ role RoleGroup. This role is used to group OWL exists restrictions together (see [20] for more details on role groups), and essentially has in this context the same effect as the earlier axiom describing the causative agent of a Penicillin allergy.

\section{Clinical Statements in the CDA}

The HL7 Clinical Document Architecture, Release 2 (CDA) [3] is a standard that prescribes the structure and semantics of document markup for clinical documents such as discharge reports and progress reports. The CDA is a derivation of the HL7 Reference Information Model (RIM) [2] which enables it to refer to external code systems such as SNOMED CT ${ }^{\circledR}$. CDA documents are particular instances conforming to this $\mathrm{CDA}$ and can contain text, images, and referrals to particular codes in HL7-endorsed

Table 1 SNOMED CT ${ }^{\circledR}$ Fragment Amoxicillin

\begin{tabular}{ll}
\hline (1) & Amoxicillin Tablet Has active ingredient Amoxicillin \\
$(2)$ & Amoxicillin Is a Aminopenicillin \\
$(3)$ & Aminopenicillin Is a Penicillin \\
$(4)$ & PCNAllergy Causative agent Penicillin \\
\hline
\end{tabular}


Table 2 OWL Representation SNOMED CT ${ }^{\circledR}$ Fragment Amoxicillin

(1) AmoxicillinTablet SubClassOf (HasActiveIngredientsome Amoxicillin)

(2) Amoxicillin SubClassOf Aminopenicillin

(3) Aminopenicillin SubClassOf Penicillin

(4) PCNAllergy SubClassOf (RoleGroupsome (causativeAgent some Penicillin))

code systems such as SNOMED CT ${ }^{\circledR}$. In this work, we focus on a particular fragment of the CDA, the Clinical Statement pattern, which specifies the structure and semantics of typically used RIM Acts in CDA documents such as Observation, SubstanceAdministration, Supply, Procedure, Encounter, Organizer, and Act.

Consider the following fragment of a CDA document from [4]:

1 <observation classCode="OBS" moodCode="EVN">

2 <code code="ASSERTION" codeSystem="2.16.840.1.113883.5.4" /

$>$

3 <text>Allergy to PCN manifesting as hives </text>

4 <value xsi:type="CD" code="106190000|Allergy|:246075003|

Causative agent $|=373270004|$ Penicillin - class of antibiotic -

(substance)" codeSystem="2.16.840.1.113883.6.96"/>

5 <actRelationship typeCode="MFST "inversionInd="true"

contextConductionInd="true" >

6 <observation classCode="OBS" moodCode="EVN" >

7 <code code="ASSERTION" codesys -

tem $=" 2.16 \cdot 840 \cdot 1 \cdot 113883 \cdot 5 \cdot 4 " />$

$8 \quad<$ value xsi:type="CD" code="247472004| Hives|"

codesystem="2.16.840.1.113883.6.96">

$9 \quad<$ displayName value="Hives" / >

$10</$ value $>$

$11</ o b s e r v a t i o n>$

$12</$ actRelationship>

$13</$ observation>

Without going into details (see [3] for more on CDA documents) the fragment consists of an Observation of an allergy to penicillin manifesting as hives. It has moodCode $=" E V N "$ indicating that the observation has occurred and has a particular code indicating that the observation is an ASSERTION, where ASSERTION is in turn a code from the code system 2.16.840.1.113883.5.4, the HL7 ActCode code system.

The value of the observation is taken from the code system SNOMED $\mathrm{CT}^{\circledR}$, identified by 2.16 .840 .1 .113883 .6 .96 , and is of data type CD, an HL7 defined data type that allows for the combination of codes from a code system to define specific concepts. Indeed, the value uses different codes from SNOMED $\mathrm{CT}^{\circledR}$ to describe the so-called post-coordinated expression 106190000| Allergy| :246075003|Causative agent $|=373270004|$ Penicillin - class of antibiotic - (substance), an allergy that has penicillin as a causative agent.

The actRelationship on line 5 with typeCode="MFST" relates this observation subsequently with the observation on line 6 , its manifestation. This observation is similarly defined as an ASSERTION with a value code from SNOMED CT ${ }^{\mathbb{B}}$ that represents hives. 


\section{Guidelines on Using SNOMED CT ${ }^{\circledR}$ in HL7 CDA Documents}

The use of external code systems such as SNOMED CT ${ }^{\circledR}$ in HL7 specifications is one aspect of achieving the HL7 goal of semantic interoperability: not only can different parties understand clinical information syntactically, they also agree on the meaning of this clinical information. By committing to widespread ontologies such as SNOMED $\mathrm{CT}^{\circledR}$, each party knows what is meant when using particular SNOMED $\mathrm{CT}^{\circledR}$ terminology.

However, when representing clinical information conforming to a reference model like the HL7 RIM, just using SNOMED CT ${ }^{\mathbb{B}}$ codes is not sufficient to avoid semantic mismatches. For example, the HL7 CDA Observation class has 2 attributes, Observation.code and Observation.value: how to use these attributes to represent clinical findings, i.e., results of a clinical observation, such as has a fracture of her left femur? One could use the code attribute to encode this statement via a SNOMED $\mathrm{CT}^{\circledR}$ expression, but it would be unclear how the value attribute should be assigned, and vice versa.

The set of guidelines presented in Using SNOMED CT in HL7 Version 3; Implementation Guide, Release 1.5 [4] addresses issues like the above: how to use SNOMED $\mathrm{CT}^{\mathbb{B}}$ in the context of HL7 clinical statement patterns? Clinical statement patterns [21] are constraints on the HL7 RIM and occur in the CDA. In the example above, the code for the observation can be an ASSERTION and the value can be a SNOMED CT ${ }^{\circledR}$ expression for has a fracture of her left femur which is a subexpression of the clinical finding SNOMED CT ${ }^{\circledR}$ concept.

We will focus in this paper on the guidelines around the SNOMED $C T^{\circledR}$ vocabulary domain constraints [[4], Section 5] and this in context of the clinical statement pattern in the CDA. Note that the approach we present here can be applied to clinical statement patterns in general.

Consider a constraint from [[4], Section 5] in Table 3. It indicates that for Observation classes with class code $O B S$, and if a SNOMED $\mathrm{CT}^{\circledR}$ expression is used for the Observation.value (this is not explicitly present in Table 3. It is, however, a prerequisite for the constraints to be applicable) and the Observation.code is ASSERTION, then the Observation.value should be a subclass of the SNOMED $\mathrm{CT}^{\circledR}$ concept Clinical Finding

Table 3 Constraint on Observation.value when Observation.code is ASSERTION

\begin{tabular}{|c|c|}
\hline Class Name & Observation \\
\hline Class Code & OBS \\
\hline Attribute Name & Observation.value \\
\hline $\begin{array}{l}\text { Narrative } \\
\text { description }\end{array}$ & $\begin{array}{l}\text { An act that is intended to result in new information about a subject. The main difference } \\
\text { between observations and other acts is that it has a value attribute that is used to state } \\
\text { the result of the assessment action described in Act.code. }\end{array}$ \\
\hline $\begin{array}{l}\text { Simple } \\
\text { representation }\end{array}$ & $\begin{array}{l}\text { ( }<<404684003 \mid \text { clinical finding|) OR }(<<413350009 \mid \text { finding with } \\
\text { explicit context|)OR }(<<272379006 \mid \text { event|)) }\end{array}$ \\
\hline Notes & $\begin{array}{l}\text { Where Observation.code = ASSERTION. } \\
\text { An alternative approach (now deprecated) is for the same value set to be communicated } \\
\text { in Observation.code where the attribute Observation.value is not present in the } \\
\text { Observation class instance. } \\
\text { As indicated in section } 2.2 .2 .2 \text { subheading 7, the situation may arise in which Observation. } \\
\text { value is a SNOMED CT expression from the set specified in the 'simple representation' } \\
\text { field of this table and Act.code is represented by a code other than "ASSERTION". For } \\
\text { such an approach can only be safely used if interpretation of the Act.code together with } \\
\text { the Observation.value does not yield a meaning that is substantially different from the } \\
\text { meaning implied if the Act.code was "ASSERTION". Without exhaustive scrutiny of } \\
\text { SNOMED CT'S content it is not possible to identify that set of codes that can safely be } \\
\text { used in this way in Act.code. }\end{array}$ \\
\hline
\end{tabular}


or of Finding with Explicit Context or of Event. The ' $<<$ ' in front of a SNOMED CT ${ }^{\circledR}$ expression represents all concepts that are subclasses of that expression or the expression itself.

\section{Used Method}

We show how to verify a given CDA document with respect to the guidelines for the use of SNOMED $\mathrm{CT}^{\circledR}$, in other words, we test if the CDA document satisfies these guidelines?

As illustrated in Figure 1 our method consists of transforming, by means of an XSL transformation, a particular CDA document in XML syntax to a set of OWL individuals using a CDA ontology and the SNOMED CT ${ }^{\circledR}$ OWL representation. We further write the constraints on the use of SNOMED CT ${ }^{\circledR}$ from [[4], Section 5] as OWL axioms and then test the consistency of those OWL axioms with respect to the set of OWL individuals that represent the original CDA document.

\section{Constructing the CDA Ontology}

In order to write CDA documents as particular OWL individuals, specifically for testing CDA documents for conformance to the SNOMED $\mathrm{CT}^{\circledR}$ guidelines, we define an ontology representing the relevant parts of the clinical statement pattern of the CDA. Based on the RIM in Figure three of [3], we model, for example, the CDA Observation class as in Table 4.

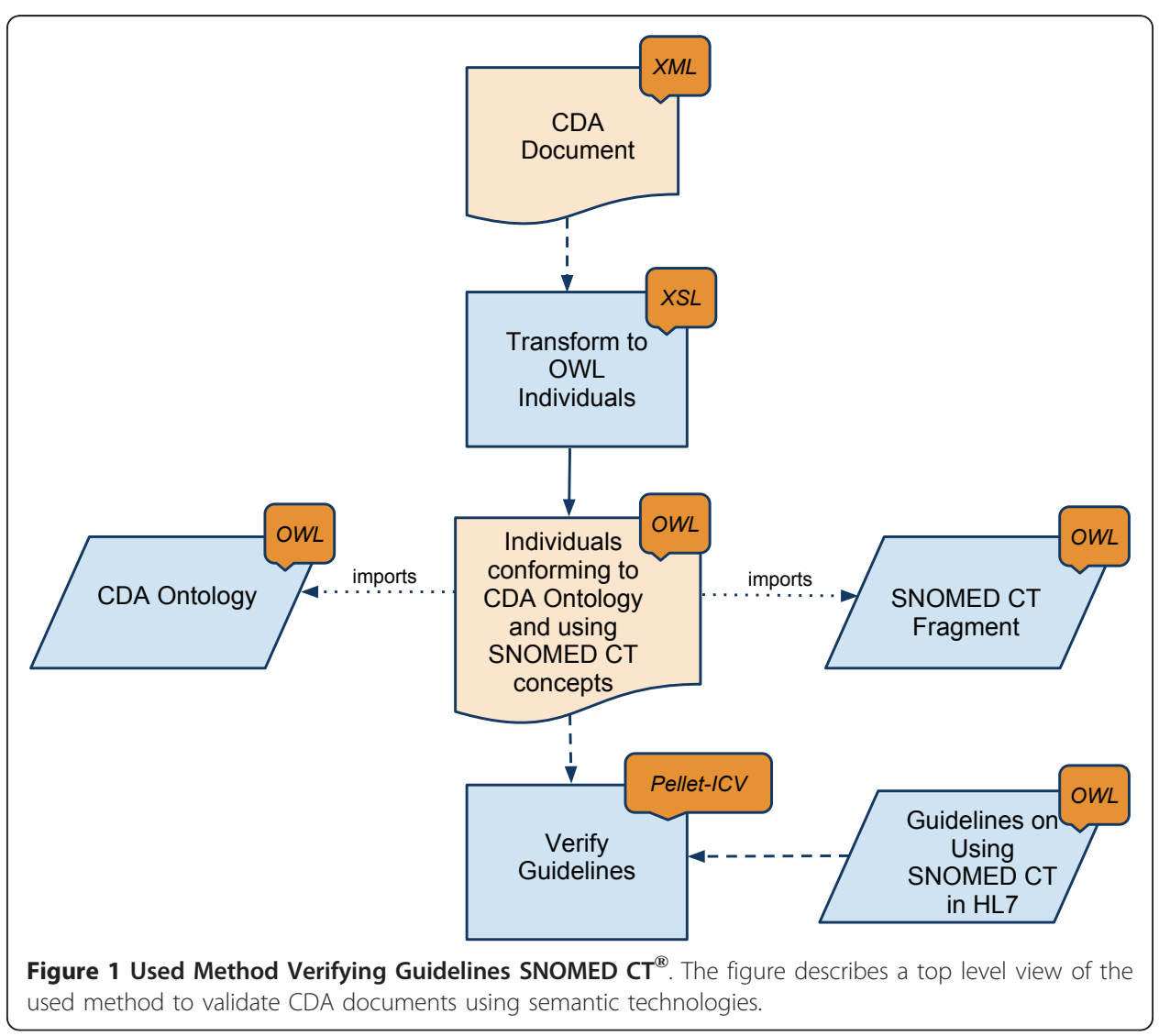


Table 4 OWL Representation of the CDA Observation Class

\begin{tabular}{ll}
\hline (1) Observation SubClassOf classCode some OBS \\
(2) Observation SubClassOf classCode only OBS \\
(3) Observation SubClassOf moodCode some $x$ _ActMoodDocumentObservation \\
(4) Observation SubClassOf moodCode only X_ActMoodDocumentObservation \\
(5) Observation SubClassOf hasValue only HL7SupportedCodeSystems or HL7ValueSets \\
(6) & Observation SubClassOf hasCode only HL7SupportedCodeSystems or HL7ValueSets \\
\hline
\end{tabular}

We note the use of the concepts HL7SupportedCodeSystems and HL7ValueSets in axioms (5) and (6). They indicate that CDA Observations have values and codes that come either from an HL7 supported code system or from an HL7 value set. An example of such an HL7 supported code system is SNOMED CT ${ }^{\circledR}$ or the HL7 ActClass code system. Indeed, besides the classes appearing in the CDA clinical statement pattern, we also add to this ontology the particular HL7 supported code systems with their hierarchically organized codes and the HL7 value sets that are defined using those codes. For example, Table 5 describes the concept code $O B S$ as a subclass of the concept code $A C T$, a subclass of the code system ActClass that is in turn a subclass of the placeholder concept HL7SupportedCodeSystems.

The value sets are defined as hierarchies as well and subclass the placeholder concept HL7ValueSets. They are defined using concepts that subclass from HL7SupportedCodeSystems as can be seen in a fragment in Table 6. There, $x \_$ActMoodDocumentObservation is defined as equivalent to any of the concept codes in HL7 supported code systems on the right-hand side of axiom (1). It additionally is indicated to be a subclass of the placeholder for value sets using the coding system ActMood which in turn is a subclass of the placeholder concept HL7ValueSets.

Reconsidering Table 4, one sees that axiom $(1,2)$ and $(3,4)$ indicate that CDA Observations should have a class code that is of type $O B S$ and a mood code that is of type $x_{-}$ActMoodDocumentObservation respectively. Axiom (1) in Table 6 indicates that a mood code that is of type $E V N$ would thus be allowed as the mood code for CDA Observations.

The used CDA ontology can be found as Additional file 1 or at http://stijnheymans. net/ontologies/CDA_ClinicalStatement_for_TermInfo.txt.

\section{Transforming the CDA Document to OWL Individuals}

Reconsider the earlier XML fragment of the CDA document. Note the presence of 2 CDA Observations, each with a class code OBS, a mood code EVN, a code, and a value. For each of those 2 observations, we introduce 2 OWL individuals and we use the previously introduced CDA ontology to define their class and mood code, as well as the code and value for those observations. Table 7 lists the OWL individuals for the first observation.

Note the definition of the individual obs_pcn_allergy that is of type Observation (a concept from the CDA ontology), and is related via the role classCode (also defined in

Table 5 HL7 Supported Code Systems Fragment

\begin{tabular}{ll}
\hline (1) & OBS SubClassOf ACT \\
(2) & ACT SubClassOf ActClass \\
(3) & ActClass SubClassOf HL7SupportedCodeSystems \\
\hline
\end{tabular}


Table 6 HL7 Value Sets Fragment

(1) $\quad x_{-}$ActMoodDocumentObservation EquivalentTo DEF or EVN or GOL or INT or PRMS or PRP or RQO

(2) $\quad x \_$ActMoodDocumentObservation SubClassOf VSUsingActMood

(3) VSUsingActMood SubClassOf HL7ValueSets

the CDA ontology) to the individual class_code_obs1 that represents the class code. The individuals class_code_obs1, mood_code_obs1, code_obs1, and value_obs1 are in turn defined appropriately using the CDA ontology as in Table 8.

Note that the concept ASSERTION is a concept code in the HL7 ActCode code system where the latter is an HL7 supported code system and thus present in our defined CDA ontology. Further note how the post-coordinated expression $106190000 \mid$ Allergy|:246075003|Causative agent|=373270004| Penicillin class of antibiotic - (substance) was rewritten as the OWL expression using the RoleGroup role and concepts from the SNOMED $\mathrm{CT}^{\circledR}$ OWL ontology: Allergy and (RoleGroupsome (causativeAgentsome Penicillin)).

The use of OWL expressions that are constructed using SNOMED $\mathrm{CT}^{\circledR}$ concepts implies that to write a CDA document as a set of individuals, we import not only the CDA ontology but also the SNOMED CT ${ }^{\circledR}$ OWL ontology. We further correctly connect SNOMED CT ${ }^{\circledR}$ with the CDA ontology by making the top SNOMED CT ${ }^{\circledR}$ concept 138875005 |SNOMED CT Concept (SNOMED RT+CTV3) a subclass of the placeholder concept SNOMEDClinicalTerms (in turn a subclass of the HL7SupportedCodeSystems concept) in the CDA Ontology.

The reader can find the used fragment of SNOMED $\mathrm{CT}^{\circledR}$ in Additional file 2 or at http://stijnheymans.net/ontologies/SNOMED_CT_for_TermInfo.txt.

Note that we do not model the actRelationship as the SNOMED $\mathrm{CT}^{\circledR}$ guidelines are scoped to isolated CDA classes. As such the OWL translation of a CDA document is not lossless: we lose the structure of the CDA documents, which, on the other hand, causes the OWL individuals ontology to have a simple at structure.

\section{The Guidelines on Using SNOMED CT ${ }^{\circledR}$ in HL7 CDA Documents as OWL Integrity Constraints}

We need one more component to be able to verify CDA documents for conformance with the guidelines on using SNOMED $\mathrm{CT}^{\circledR}$ : the guidelines written as OWL Integrity Constraints.

Take, for example, the constraint expressed in Table 3, which we can phrase as

If an Act has a class code OBS (i.e., it is an Observation), and the Observation's code is ASSERTION, and SNOMED CT ${ }^{\circledR}$ is used to provide a value for the Observation, then this possibly post-coordinated expression has to be either a 404684003

Table 7 OWL Individuals for CDA Observations

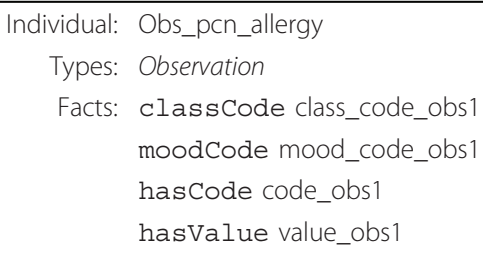


Table 8 OWL Individuals

\begin{tabular}{cl}
\hline Individual: & class_code_obs1 \\
Types: & OBS \\
\hline Individual: & mood_code_obs1 \\
Types: & EVN \\
\hline Individual: & Code_obs1 \\
Types: & ASSERTION \\
\hline Individual: & value_obs1 \\
Types: & Allergy and \\
& (RoleGroupsome (causativeAgentsome Penicillin)) \\
\hline
\end{tabular}

|clinical finding or a 413350009 |finding with explicit context or a 272379006 | event.

We write this constraint as the following OWL integrity constraint:

$$
\begin{aligned}
& \text { (valueof some (Observation and } \\
& \text { (hasCode some ASSERTION)) ) and } \\
& \text { (codesystem value 2.16.840.1.11388.3.6.96) } \\
& \text { SubClassOf } \\
& \text { ClinicalFinding or FindingWithContext or Event }
\end{aligned}
$$

The first component of this integrity constraint, (valueofsome (Observation and (hasCodesome ASSERTION ))), picks up the individuals that are the value of some CDA Observation (valueof is the inverse of hasValue in the CDA ontology) where the CDA Observation has a code that is an ASSERTION. These individuals additionally have to be related via the codeSystem role to the individual 2.16.840.1.11388.3.6.96 which represents the SNOMED CT ${ }^{\circledR}$ code system. The SubClassOf then indicates that such individuals need to be either of type ClinicalFinding, FindingWithContext, or Event.

The other constraints in [[4], Section 5] can be written similarly as OWL integrity constraints. The reader can find the integrity constraints in Additional file 3 or at http://stijnheymans.net/ontologies/TermInfo_ICs.txt.

\section{Verification of the Guidelines}

Reconsider the example CDA document in Tables 7 and 8. Is this document satisfied by the constraints on the use of SNOMED $\mathrm{CT}^{\circledR}$ ?

Take, for example, the integrity constraint (4). The individual value_obs1 is the valueof obs_pcn allergy which is of type Observation. Indeed, valueof is the inverse role of hasValue and obs_pcn_allergy hasValue value_obs1. Moreover, the individual obs_pcn_allergy has a code code_obs1 that is of type ASSERTION. Finally, since value_obs1 is defined using concepts from the SNOMED CT ${ }^{\circledR}$ ontology it inherits the codeSystem value 2.16.840.1.11388.3.6.96 from the placeholder concept SNOMEDClinicalTerms for SNOMED CT ${ }^{\circledR}$ in the CDA ontology. Note that the ontology with OWL individuals corresponding to the CDA document imports both the CDA ontology and the SNOMED $\mathrm{CT}^{\circledR}$ ontology and it appropriately makes the top SNOMED CT ${ }^{\circledR}$ concept a subclass of SNOMEDClinicalTerms in the CDA ontology. 
The individual value_obs1 thus satisfies the left-hand side of integrity constraint (4) - left/right is with respect to the SubClassOf symbol. In order for the integrity constraint to be satisfied the individual value_obs1 is required to also satisfy the righthand side, i.e., it has to be of type ClinicalFinding or FindingWithContext or Event. Recall from Table 8 that value_obs1 is of type Allergy and (RoleGroupsome (causativeAgentsome Penicillin)).

As the OWL individuals corresponding to the CDA document import the SNOMED $\mathrm{CT}^{\circledR}$ OWL ontology, we can deduce with standard OWL reasoning that Allergy and (RoleGroupsome (causativeAgentsome Penicillin)) is a subclass of the concept ClinicalFinding, and thus, that value_obs1 is of type ClinicalFinding, and finally, also of ClinicalFinding or FindingWithContext or Event. This satisfies the integrity constraint corresponding to the SNOMED CT ${ }^{\circledR}$ guideline.

Note that we intertwine standard OWL reasoning with OWL Integrity Constraint reasoning: we use standard reasoning to infer concepts in SNOMED $\mathrm{CT}^{\circledR}$ and use the Integrity Constraint semantics to test the constraints. To illustrate this, assume we replace the integrity constraint (4) with the integrity constraint (5) where we leave out the clinical finding concept on the right-hand side:

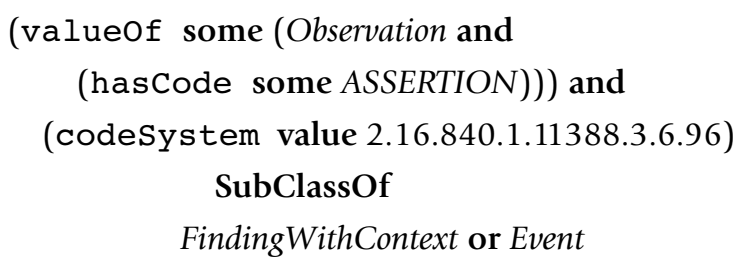

Again we have that value_obs1 satisfies the left-hand side of the integrity constraint. As before, and using standard OWL semantics, we can infer that value obs1 is of type ClinicalFinding. However, to satisfy the integrity constraint, we would need that it is of type FindingWithContext or Event. If we would use the standard OWL semantics we would infer that value_obs1 is indeed of that type in order to satisfy the integrity constraint. Using the integrity constraint semantics, the constraint is violated as we cannot infer via SNOMED $\mathrm{CT}^{\circledR}$ (which we treat with standard OWL semantics) that it is of the desired type.

\section{Implementation}

We implemented the used method to demonstrate the validation of CDA documents in terms of guidelines on the use of SNOMED CT ${ }^{\circledR}$. Our implementation is based on the Open Health Workbench [7], an Eclipse-based tool for editing HL7 clinical documents. We added our technology to the Open Health Workbench in the form of an Eclipse plug-in. Consequently, we can open HL7 CDA documents and via a newly added validation option, we can automatically check SNOMED $\mathrm{CT}^{\mathbb{R}}$ usage guidelines for this document. The output of such a violation indicates which of the HL7 guidelines was violated and assists the user in fixing the CDA document such that it does conform to the guidelines.

Under the hood, as explained above, we work with OWL as the lingua franca for our validation. As SNOMED CT ${ }^{\circledR}$ provides an OWL version and we designed OWL integrity constraints for the guidelines on using SNOMED $\mathrm{CT}^{\circledR}$ in CDA documents, the only remaining component for automatic transformation to OWL is the CDA 
document. We accomplish the automatic transformation of CDA documents by using an XSL transformation that takes the XML format supported by the Open Health Workbench and translates this on the fly to OWL individuals conforming to the CDA ontology. The actual validation incorporating the different OWL ontologies is then done using Pellet-ICV [16].

A demo demonstrating the technology is available [22].

\section{Results and Discussion}

We tested our method on an archive of existing CDA document examples provided in [23]. A typical violation of the guidelines of using SNOMED CT ${ }^{\circledR}$ in CDA documents can be seen in the below fragment from HITSP C32: HITSP Summary Documents Using HL7 CCD at [24]:

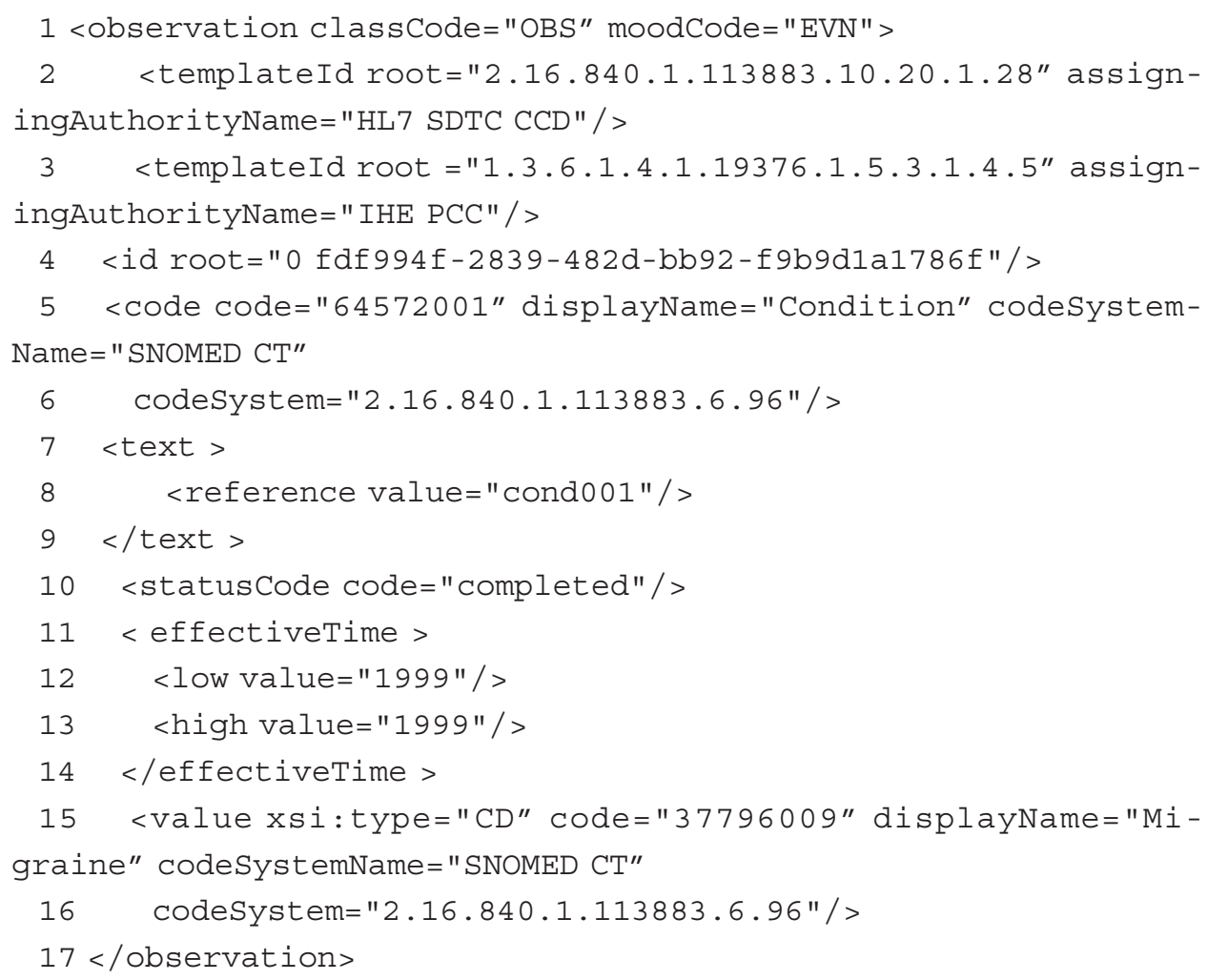

Indeed, we have in [[4], Section 5.3.1.1] a vocabulary domain constraint saying that an Ob-servation.value should be $((<<281296001 \mid$ result comments $\mid)$ OR $(<<260245000 \mid$ findings values $\mid))$ if SNOMED CT ${ }^{\circledR}$ is used to encode the value. This constraint is violated as Migraine is neither a subtype of the result comments concept nor of the findings values concept. Actually, the Migraine is a subtype of the used Observation.code Condition.

Additionally, this fragment also violates the constraint saying that an Observation. code should be ( $(<<386053000 \mid$ evaluation procedure|) OR $(<<363787002$ |observable entity|)), as Condition is neither an observable entity nor an evaluation procedure.

A possible way of resolving this issue is to use ASSERTION for the Observation.code. The Observation.value can then be left as is. 
As a proof-of-concept, we further analyzed $26 \mathrm{CDA}$ documents that are available from [23]. Note that only 14 of those 26 documents use SNOMED CT ${ }^{\circledR}$ concepts in their definition, such that they are the only relevant ones for analysis. We grouped the 8 Integrity Constraints that validate the usage of SNOMED CT ${ }^{\circledR}$ in the documents in 5 classes pertaining to Observations, Entities, Procedures, Substance Administrations, and Organizers. The group of Observation constraints contains 3 axioms while the other groups contain 1 violation constraint each. Table 9 shows the 5 groups in the left-hand column. The second column lists how many statements in the 14 CDA documents refer to either Observations, Entities, Procedures, Substance Administrations, Supplies, and Organizers by means of SNOMED CT ${ }^{\circledR}$ concepts. The third column indicates how many of those statements were violated by the particular group of Integrity Constraints. The final column shows the percentage of violations.

We note that even though the sample is not significant, $73 \%$ of the statements that use Observations using SNOMED $\mathrm{CT}^{\circledR}$ are violated. The CDA documents do not make extensive use of other definitions than Observations, so other results are not indicative. Note that the example is typical for the violated constraints in this $73 \%$ group around Observations.

A remaining challenge in our approach is the usage of the full SNOMED CT ${ }^{\circledR}$ ontology. Due to its sheer size this is at the moment practically impossible. In our examples as well as the prototype, we use a tailored SNOMED CT ${ }^{\circledR}$ ontology that incorporates the relevant hierarchies based on the CDA document we want to validate. The tailored version defines 358 concepts and is thus significantly smaller than the full SNOMED $\mathrm{CT}^{\circledR}$ which contains close to 300,000 concepts. It is part of future research to extract the relevant SNOMED $\mathrm{CT}^{\circledR}$ fragment for which we will consider modular extraction techniques [25].

The current prototype supports transformation of CDA documents to OWL individuals where the CDA document contains only simple SNOMED $\mathrm{CT}^{\circledR}$ concepts: postcoordinated expressions are not translated to their proper OWL equivalents yet. Additionally, the prototype currently uses Pellet-ICV to validate OWL Integrity Constraints. As the latter is closed source software, it is not ideal as a final solution. We are working on both issues: the translation of SNOMED $\mathrm{CT}^{\circledR}$ expressions confirming to the compositional grammar to proper OWL and the usage of alternatives for Pellet ICV such as DL-programs [26] as described in [16].

Besides the SNOMED CT ${ }^{\circledR}$ vocabulary domain constraints in [[4], Section 5], the implementation guide [4] provides also guidelines on the overlaps between the RIM and SNOMED CT ${ }^{\circledR}$ semantics [[4], Section 2]. These overlaps are in general much harder, if possible at all, to model in OWL. Guidelines such as

Table 9 Initial Evaluation Results

\begin{tabular}{lccc}
\hline & SNOMED CT $^{\circledR}$ Usages in CDA & Violated Statements & $\%$ \\
\hline Observation & 239 & 175 & $73 \%$ \\
Entity & 6 & 0 & $0 \%$ \\
Procedure & 7 & 0 & $0 \%$ \\
Substance Administration & 2 & 2 & $100 \%$ \\
Organizer & 4 & 2 & $50 \%$ \\
\hline
\end{tabular}


"Act class clones SHALL include the priorityCode attribute if there is a requirement for expressing the urgency of a request, tracking and auditing services based on requested prioritization or any other aspects of workflow management related to priority."

are largely dependent on human intervention. Only a human can decide whether the goal is to express the urgency of a request. We intend to model as much as possible of [[4], Section 2] as integrity constraints, similarly to our treatment of all constraints in [[4], Section 5], and thus identifying a subset of the constraints that are expressible in OWL. This would provide a sense of focus for CDA document developers: which guidelines can be handled automatically, which ones need special manual care?

\section{Conclusions}

We showed a strategy enabling automatic validation of the implementation guidelines/ rules on using SNOMED $\mathrm{CT}^{\circledR}$ in HL7 documents. Using the available SNOMED CT ${ }^{\circledR}$ OWL representation and a Clinical Statement OWL representation, one can use OWL Integrity Constraints to automatically validate $\mathrm{CDA}$ documents regarding their conformance with the vocabulary domain constraints in [[4], Section 5]. As such it removes the burden from IT professionals of having to manually implement such guidelines in systems that use HL7 Version 3 documents.

\section{Additional material}

Additional file 1: CDA Clinical Statement Ontology for Termlnfo Guidelines.

Additional file 2: SNOMED CT ${ }^{\circledR}$ Fragment Used in Prototype.

Additional file 3: Termlnfo Constraints.

\section{Acknowledgements}

This material includes SNOMED Clinical Terms ${ }^{\circledR}\left(\right.$ SNOMED $\left.C T^{\circledR}\right)$ which is used by permission of the International Health Terminology Standards Development Organisation (IHTSDO). All rights reserved. SNOMED CT ${ }^{\circledR}$, was originally created by The College of American Pathologists. "SNOMED" and "SNOMED CT" are registered trademarks of the IHTSDO.

\section{Authors' contributions}

SH and JP devised the described used method. SH prepared a draft of the article and JP did major revisions. MM implemented the plug-in for the Open Health Workbench, scripted and built the demo, and contributed to revisions of the paper. All authors read and approved the final manuscript.

\section{Competing interests}

The authors declare that they have no competing interests.

Received: 14 October 2010 Accepted: 15 July 2011 Published: 15 July 2011

\section{References}

1. Health Level Seven International. [http://www.hl7.org/].

2. George Beeler J, Duteau JH, Grieve G, McKenzie L, Nelson D: HL7 Reference Information Model. 2010 [http://www.hl7. org/, Version 0231].

3. Dolin RH, Alschuler L, Boyer S, Beebe C, Behlen FM, Biron PV, Shabo A: HL7 Clinical Document Architecture, Release 2. Journal of the American Medical Informatics Association 2006, 13:30-39.

4. Cheetham E, Markwell D, Dolin R: Using SNOMED CT in HL7 Version 3; Implementation Guide, Release 1.5. 2009 [http://www.hl7.org/], [HL7 Version 3 Implementation Guide: Using SNOMED CT, Release 1 Last Ballot: DSTU Ballot 4].

5. Patel-Schneider PF, Hayes P, Horrocks I: OWL Web Ontology Language Semantics and Abstract Syntax. Recommendation 10 February 2004, W3C 2004 [http://www.w3.org/TR/owl-semantics/].

6. Uschold M, Grüninger M: Ontologies: principles, methods, and applications. Knowledge Engineering Review 1996, 11(2):93-155.

7. Open Health Workbench. [https://www.projects.openhealthtools.org/sf/projects/openhealthworkbench/]. 
8. Berners-Lee T, Hendler J, Lassila O: The Semantic Web. Scientific American 2001, 284(5):34-43.

9. Klyne G, Carroll JJ: Resource Description Framework (RDF): Concepts and Abstract Syntax. Recommendation 10 February 2004, W3C 2004 [http://www.w3.org/TR/rdf-concepts/].

10. Brickley D, Guha RV: RDF Vocabulary Description Language 1.0: RDF Schema. Recommendation 10 February 2004, W3C 2004 [http://www.w3.org/TR/rdf-schema/].

11. Motik B, Patel-Schneider PF, Parsia B: OWL 2 Web Ontology Language Structural Specification and Functional-Style Syntax. Recommendation 27 October 2009, W3C 2009 [http://www.w3.org/TR/owl2-syntax/].

12. Baader F, Calvanese D, McGuinness DL, Nardi D, Patel-Schneider PF, (Eds): The Description Logic Handbook Cambridge University Press; 2003.

13. Spackman K, Campbell K, Côté R: SNOMED RT: A reference terminology for health care. In AMIA'97- Proceedings of the 1997 AMIA Annual Fall Symposium. Edited by: Masys D. Philadelphia: Hanley 1997:640-644

14. The Manchester OWL Syntax. [http://www.co-ode.org/resources/reference/manchester_syntax/].

15. Tao J, Sirin E, Bao J, McGuinness D: Integrity Constraints in OWL. Proceedings of the Twenty-Fourth AAAl Conference on Artificial Intelligence (AAAI 2010) AAAI Press; 2010.

16. Pellet Integrity Constraints: Validating RDF with OWL. [http://clarkparsia.com/pellet/icv/]

17. Pellet: OWL 2 Reasoner for Java. [http://clarkparsia.com/pellet/]

18. International Health Terminology Standards Development Organisation - SNOMED CT. [http://www.ihtsdo.org/ snomed-ct/].

19. Spackman K: SNOMED CT Stated Relationships Guide. International Health Terminology Standards Development Organisation 2010, [Tabular Format and OWL Transformations].

20. Spackman KA, Dionne R, Mays E, Weis J: Role Grouping as an Extension to the Description Logic of Ontylog, motivated by Concept Modeling in SNOMED. Proc of AMIA Symp 2002, 712-716.

21. Bishop C, Buitendijk H, Frean I, Loyd P, Smithies R: Domain: Clinical Statement. 2010 [http://www.hl7.org/], [HL7 Version 3 Standard: Clinical Statement Pattern, Release 1 Last Ballot: Draft for Comment Ballot 1].

22. Validation of SNOMED CT Terms in HL7 CDA Documents. [http://demo.semanticbits.com/public/videos/ CDAValidation/CDAValidation.htm].

23. Spronk R: CDA Around the World: Archive of CDA Instance Examples.[http://www.ringholm.de/download/ CDA_R2_examples.zip], [Accessed July 2010].

24. NIST: CDA Guideline Validation.[http://xreg2.nist.gov/cda-validation/downloads.html], [Accessed July 2010].

25. Konev B, Lutz C, Walther D, Wolter F: CEX and MEX: Logical Diff and Logic-based Module Extraction in a Fragment of OWL. OWL: Experiences and Directions (OWLED) 2008

26. Eiter T, lanni G, Lukasiewicz T, Schindlauer R, Tompits H: Combining Answer Set programming with Description Logics for the Semantic Web. Artificial Intelligence 2008, 172(12-13):1495-1539.

doi:10.1186/2041-1480-2-2

Cite this article as: Heymans et al: Semantic validation of the use of SNOMED CT in HL7 clinical documents. Journal of Biomedical Semantics 2011 2:2.

\section{Submit your next manuscript to BioMed Central and take full advantage of:}

- Convenient online submission

- Thorough peer review

- No space constraints or color figure charges

- Immediate publication on acceptance

- Inclusion in PubMed, CAS, Scopus and Google Scholar

- Research which is freely available for redistribution 